\title{
SSTR-RADS Version 1.0 as a Reporting System for SSTR PET Imaging and Selection of Potential PRRT Candidates: A Proposed Standardization Framework
}

\author{
Rudolf A. Werner ${ }^{1-3}$, Lilja B. Solnes ${ }^{1}$, Mehrbod S. Javadi ${ }^{1}$, Alexander Weich ${ }^{3,4}$, Michael A. Gorin ${ }^{1,5}$, \\ Kenneth J. Pienta ${ }^{5}$, Takahiro Higuchi ${ }^{2,6,7}$, Andreas K. Buck ${ }^{2,3,6}$, Martin G. Pomper ${ }^{1,5}$, Steven P. Rowe*1,5, and \\ Constantin Lapa*2,3 \\ ${ }^{I}$ Division of Nuclear Medicine and Molecular Imaging, The Russell H. Morgan Department of Radiology and Radiological Science, \\ Johns Hopkins University School of Medicine, Baltimore, Maryland; ${ }^{2}$ Department of Nuclear Medicine, University Hospital \\ Würzburg, Würzburg, Germany; ${ }^{3}$ NET Center Würzburg, European Neuroendocrine Tumor Society (ENETS) Center of Excellence \\ (CoE), University Hospital Würzburg, Würzburg, Germany; ${ }^{4}$ Department of Internal Medicine II, Gastroenterology, University \\ Hospital Würzburg, Würzburg, Germany; ${ }^{5}$ James Buchanan Brady Urological Institute and Department of Urology, Johns Hopkins \\ University School of Medicine, Baltimore, Maryland; ${ }^{6}$ Comprehensive Heart Failure Center, University Hospital Würzburg, \\ Würzburg, Germany; and ${ }^{7}$ Department of Bio Medical Imaging, National Cardiovascular and Cerebral Research Center, Suita, Japan
}

Reliable standards and criteria for somatostatin receptor (SSTR) PET are still lacking. We herein propose a structured reporting system on a 5-point scale for SSTR PET imaging, titled SSTR-RADS version 1.0, which might serve as a standardized assessment for both diagnosis and treatment planning in neuroendocrine tumors. SSTR-RADS could guide the imaging specialist in interpreting SSTR PET scans, facilitate communication with the referring clinician so that appropriate workup for equivocal findings is pursued, and serve as a reliable tool for patient selection for planned peptide receptor radionuclide therapy.

Key Words: ${ }^{68} \mathrm{Ga}$-DOTATATE/-TOC; SSTR; somatostatin receptor; PET; PRRT; peptide receptor radionuclide therapy; neuroendocrine tumor; RADS

J Nucl Med 2018; 59:1085-1091

DOI: 10.2967/jnumed.117.206631

\section{B} ecause of the promising results of recent randomized clinical trials, peptide receptor radionuclide therapy (PRRT) of inoperable, metastasized neuroendocrine tumors (NETs) is increasingly being used $(1,2)$. As a prerequisite for endoradiotherapy using hot somatostatin analogs (SSAs), somatostatin receptor (SSTR) PET using agonists including ${ }^{68} \mathrm{Ga}$-DOTATOC/DOTATATE and ${ }^{68} \mathrm{Ga}-$ DOTA, 1-Nal3-octreotide ( ${ }^{68} \mathrm{Ga}$-DOTANOC) or antagonists such as ${ }^{68} \mathrm{Ga}-\mathrm{NODAGA}-J R 11$ ( $\left.{ }^{68} \mathrm{Ga}-\mathrm{OPS} 202\right)$ must demonstrate sufficient radiotracer uptake in suggestive NET lesions (3-5). Hence, from a long-term perspective, increased use of SSTR PET can be envisaged. However, in contrast to the clinically well-established

Received Dec. 5, 2017; revision accepted Mar. 14, 2018.

For correspondence or reprints contact: Constantin Lapa, University Hospital Würzburg, Department of Nuclear Medicine, Oberdürrbacherstr. 6, 97080 Würzburg, Germany.

E-mail: lapa_c@ukw.de

${ }^{*}$ Contributed equally to this work.

Published online Mar. 23, 2018.

COPYRIGHT (c) 2018 by the Society of Nuclear Medicine and Molecular Imaging. 4-point Krenning scale in terms of SSTR scintigraphy $\left({ }^{111} \mathrm{In}-\right.$ pentetreotide; OctreoScan), which is mainly based on liver-versustumor uptake (6), reliable standards and criteria for SSTR PET are still lacking (7). Of note, numerous studies have reported on pitfalls in interpreting SSTR PET scans: the physiologic distribution includes tissues that potentially exhibit SSTR, such as the pituitary gland, tonsils, thyroid, adrenal glands, liver, spleen, or the head of the pancreas $(8,9)$; macrophages also express SSTR on the cell surface, and increased inflammatory activity might lead to potential false-positive radiotracer uptake $(10,11)$; individual tumors can have heterogeneous levels of SSTR expression related to their degree of differentiation (G1-G3) (7); and short- and long-acting SSA and chemotherapeutic agents could influence PET imaging due to receptor saturation and fluctuations of SSTR expression on the tumor cell surface $(3,11,12)$.

Hence, in analogy to reporting and data systems that have been described for multiple different organs (13-16) as well as for molecular imaging of prostate cancer (prostate-specific membrane antigen [PSMA]-reporting and data systems [RADS]) (17), we herein propose a structured reporting system on a 5-point scale for SSTR PET imaging, titled SSTR-RADS version 1.0, which might serve as a standardized assessment for both diagnosis and treatment planning in NET. SSTR-RADS could guide the imaging specialist in interpreting SSTR PET scans, aid communication with the referring clinician so that appropriate workup for equivocal findings is pursued, and serve as a reliable tool for patient selection for planned PRRT.

\section{PATIENT POPULATION}

Because the herein presented data comprise a retrospective analysis of routinely acquired data, the local ethic committee waived the need for further approval. All patients gave written and informed consent to the procedures, and all patients provided written informed consent for scientific analysis of the obtained data.

\section{OVERVIEW OF SSTR-RADS AND DESCRIPTION OF DIFFERENT CATEGORIES}

Table 1 gives an overview of the SSTR-RADS in its current form, version 1.0. The reporting system is mainly based on the format 


\begin{tabular}{|c|c|c|c|}
\hline SSTR-RADS & Finding & Uptake level $^{\star}$ & PRRT? $^{\dagger}$ \\
\hline 1 (benign) & $\begin{array}{l}\text { Known to be benign (confirmed by previous biopsy or } \\
\text { with pathognomonic appearance on conventional/ } \\
\text { anatomic imaging). }\end{array}$ & & \\
\hline $1 \mathrm{~A}$ & $\begin{array}{l}\text { Benign lesion, characterized by biopsy or in accordance to } \\
\text { anatomic imaging and without any abnormal uptake (Fig. 1). }\end{array}$ & 1 & Not to be considered. \\
\hline $1 \mathrm{~B}$ & $\begin{array}{l}\text { Benign lesion, characterized by biopsy or in accordance to } \\
\text { anatomic imaging but with increased (focal) uptake (e.g., } \\
\text { prostatitis, benign prostatic hyperplasia [Fig. 2], or thyroid } \\
\text { adenoma [Supplemental Fig. 1]). }\end{array}$ & $2-3$ & Not to be considered. \\
\hline 2 (likely benign) & $\begin{array}{l}\text { Soft-tissue site atypical of metastatic NET (e.g., axillary lymph } \\
\text { nodes); equivocal uptake in bone lesion atypical for NET (e.g., } \\
\text { strongly suspected to be degenerative, Fig. 3). }\end{array}$ & 1 & Not to be considered. \\
\hline 3 & $\begin{array}{l}\text { Further workup (biopsy, if sampling is possible) or follow-up (f/u) } \\
\text { imaging might be required. }\end{array}$ & & \\
\hline $3 A$ & Suggestive of, but not definitive for, NET. & $1-2$ & Not to be considered. \\
\hline & $\begin{array}{l}\text { Equivocal uptake in soft-tissue sites typical for NET metastases, } \\
\text { such as in regional lymph nodes (LNs, Fig. 4). Biopsy or initial } \\
\text { f/u imaging (SSTR PET or whole-body MRI after } 3 \text { mo) might } \\
\text { confirm diagnosis, also depending on Ki-67/grading (21). }\end{array}$ & & \\
\hline 3B & Suggestive of, but not definitive for, NET. & $1-2$ & $\begin{array}{l}\text { Single lesions: locoregional } \\
\text { procedure; increased number } \\
\text { of lesions: PRRT. }\end{array}$ \\
\hline & $\begin{array}{l}\text { Uptake in bone lesions not atypical for NET (Fig. 5). Initial f/u } \\
\text { imaging (SSTR PET or whole-body MRI f/u after } 3 \mathrm{mo} \text { ) might } \\
\text { confirm diagnosis, also depending on Ki-67/grading (21). }\end{array}$ & & \\
\hline $3 C$ & $\begin{array}{l}\text { Suggestive of an SSTR-expressing, non-NET benign tumor or } \\
\text { malignant process. }\end{array}$ & 3 & Not to be considered. \\
\hline & $\begin{array}{l}\text { Intense uptake in a site (highly) atypical for NET, for example, } \\
\text { breast uptake (Fig. 6) (20). Tissue confirmation of tumor } \\
\text { histology should be considered. }\end{array}$ & & \\
\hline $3 D$ & $\begin{array}{l}\text { High likelihood for malignant NET lesion, but negative on a } \\
\text { SSTR PET scan. }\end{array}$ & Not available & Not to be considered. ${ }^{\ddagger}$ \\
\hline & $\begin{array}{l}\text { Anatomic imaging representing lesion highly suggestive of } \\
\text { being malignant (dedifferentiated NET or another type of } \\
\text { malignancy), but demonstrating no SSTR uptake (e.g., } \\
\text { single dedifferentiated liver metastasis, Fig. } 7 \text {, or a non- } \\
\text { NET malignancy) }(6,25) .{ }^{18} \text { F-FDG PET might be of value } \\
(11,26,27) \text {. Tissue confirmation of tumor histology should } \\
\text { be considered. }\end{array}$ & & \\
\hline 4 (NET highly likely) & $\begin{array}{l}\text { Positive uptake in site typical for NET lesion but lacking definitive } \\
\text { findings on anatomic imaging. }\end{array}$ & 3 & To be considered." \\
\hline & $\begin{array}{l}\text { Intense uptake in common site typical for NET lesion, but } \\
\text { without confirmatory findings on anatomic imaging (e.g., } \\
\text { bone lesions or small LN, which is nonsuggestive on a } \\
\text { conventional CT scan, Fig. 8). Because of high sensitivity and } \\
\text { specificity of SSTR PET imaging, further confirmation by } \\
\text { biopsy might be not necessary. }\end{array}$ & & \\
\hline $\begin{array}{l}5 \text { (NET almost certainly } \\
\text { present) }\end{array}$ & $\begin{array}{l}\text { Intense uptake in site typical for NET with corresponding } \\
\text { findings on conventional imaging. }\end{array}$ & 3 & Definitely to be considered. \\
\hline & \multicolumn{3}{|l|}{$\begin{array}{l}\text { An example would be a SSTR-expressing liver lesion } \\
\text { with corresponding findings on a CT scan (Fig. 9). }\end{array}$} \\
\hline \multicolumn{4}{|c|}{$\begin{array}{l}{ }^{\ddagger} \text { Depends on grading, overall tumor burden, kidney and bone marrow function, and overall SSTR expression (e.g., G2 NET patient with entirely all } \\
\text { lesions demonstrating SSTR expression, but a single dedifferentiated lesion; a combined treatment of PRRT together with a locoregional procedure } \\
\text { could be considered) }(28,29) \text {. } \\
\text { IOOn the assumption that an SSTR PET-avid lesion in a typical distribution has a very high probability of representing NET, PRRT might be considered. }\end{array}$} \\
\hline
\end{tabular}


of the recently published PSMA-RADS version 1.0 (17), consensus findings from the clinical experience of the 2 involved centers (Johns Hopkins University School of Medicine and European Neuroendocrine Tumor Society-Center of Excellence Würzburg University), as well as an extensive search in electronic databases including PubMed (www.ncbi.nlm.nih.gov), Science Direct (www.sciencedirect.com), MEDLINE (https://www.nlm.nih.gov/bsd/pmresources. html), and Library of Congress (https://www.loc.gov). The search strategy included the following key words: "SSTR-PET" or "PRRT" or "DOTATATE" or "DOTATOC" and "Standardization", without restrictions to language or publication date. When the SSTRRADS reporting system is used, multiple different goals might be achieved: reflect the level of confidence of an imaging reader on the presence of a NET tumor lesion, provide guidance as to which lesions should be considered physiologic rather than pathologic, which test might be appropriate as a next step in the diagnostic algorithm (based on the SSTR PET findings), and the establishment of which patients might be suitable candidates for subsequent PRRT. Table 2 summarizes the uptake level on SSTR PET using a 3-point scale, which goes from score 1 (focal uptake, but lower than/equal to blood pool) through score 2 (higher than blood pool, but lower than/equal to physiologic liver uptake) to score 3 (higher than physiologic liver uptake). These categories will subsequently be referred to as "level 1," "level 2," or "level 3" uptake, respectively.

\section{SSTR-RADS-1}

SSTR-RADS-1 signifies definitively benign lesions, as known by previous biopsy or pathognomonic anatomic imaging. These lesions are further divided into SSTR-RADS-1A and SSTR-RADS-1B (Table 1). SSTR-RADS-1A indicates definitively benign findings without any abnormal uptake (normal physiologic biodistribution of an SSTR imaging agent (Fig. 1) $(18,19)$. On the other hand, SSTRRADS-1B includes those lesions, which although benign, demonstrate discernable radiotracer uptake, for example, prostatitis, benign prostatic hyperplasia (Fig. 2), or intense uptake in the thyroid (e.g., due to a known thyroid adenoma, Supplemental Fig. 1 [supplemental materials are available at http://jnm.snmjournals.org], level 2-3) which has been previously confirmed by biopsy or definitive diagnostic imaging (20). Of note, it has to be emphasized that SSTRRADS scores can be dynamic based on the workup that the patient has had done: If an intensively avid thyroid nodule is primarily classified as SSTR-RADS-3C (no previous imaging or other workup), then biopsied and found to be an adenoma, this finding would be rescored as SSTR-RADS-1B.

TABLE 2

A 3-Point Qualitative Assessment Scoring for Defining Uptake Level in an SSTR-Avid Lesion on a SSTR PET Scan

\begin{tabular}{lc}
\hline Uptake score & Relative uptake \\
\hline Level 1 & Uptake $\leq$ blood pool \\
Level 2 & $\begin{array}{c}\text { Uptake }>\text { blood pool but } \leq \\
\text { physiologic liver uptake }\end{array}$ \\
Level 3 & Uptake $>$ physiologic liver uptake \\
\hline
\end{tabular}

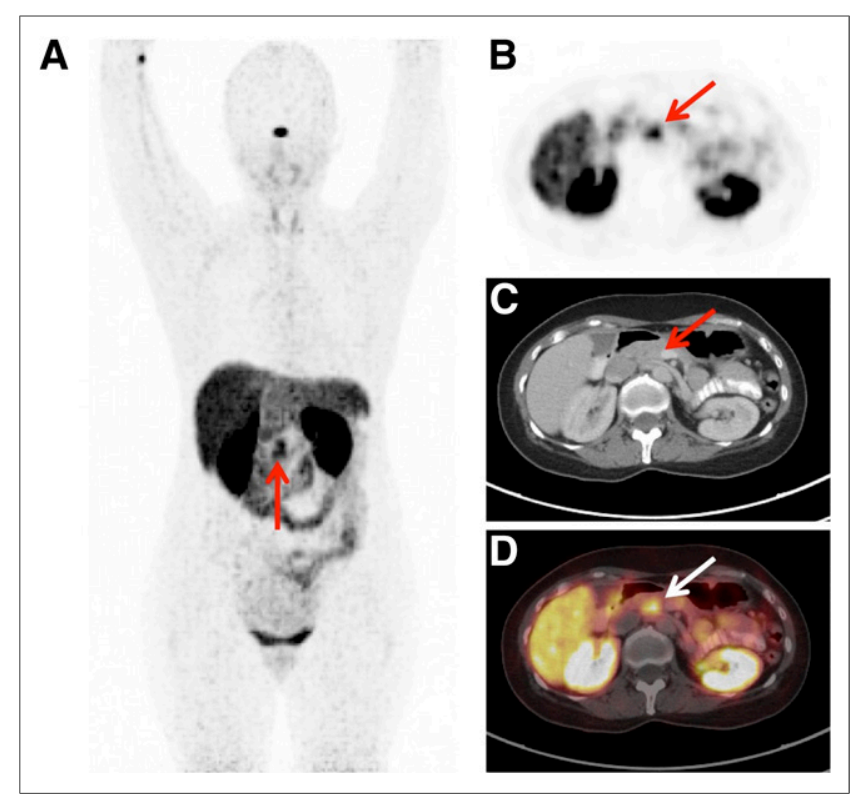

FIGURE 1. SSTR-RADS-1A. Coronal ${ }^{68} \mathrm{Ga}$-DOTATOC PET wholebody maximum-intensity projection (A) demonstrating physiologic tracer distribution. No sites of abnormal uptake can be appreciated. Normal biodistribution of agent is seen, including uptake in pituitary gland, thyroid, adrenal glands, bowel, liver, and spleen $(18,19)$. Radiotracer is excreted via urinary tract. Arrow indicates physiologic finding in uncinate process, which is also demonstrated by axial ${ }^{68} \mathrm{Ga}$-DOTATOC PET (B), axial CT (C), and axial ${ }^{68} \mathrm{Ga}-\mathrm{DOTATOC}$ PET/CT (D).

SSTR-RADS-1B might help the reader to describe a region of increased uptake but without raising a concern of malignancy (17). PRRT is definitely not considered.

\section{SSTR-RADS-2}

The SSTR-RADS-2 category describes lesions with uptake (level 1) in soft-tissue sites atypical for metastatic NET (e.g., axillary lymph nodes) or uptake in bone lesions atypical for NET (e.g., strongly suggestive of being degenerative in nature, Fig. 3). In brief, SSTR-RADS-2 includes those lesions with low levels of SSTR expression or nonspecific radiotracer uptake and that are atypical sites for NET lesions. SSTR-RADS-2 lesions are almost certainly benign; PRRT is definitely not considered.

\section{SSTR-RADS-3}

SSTR-RADS-3 includes those imaging findings that require further workup (biopsy, if sampling is possible, or follow-up imaging). Many of these lesions are suggestive of, but not definitive for, NET tumors.

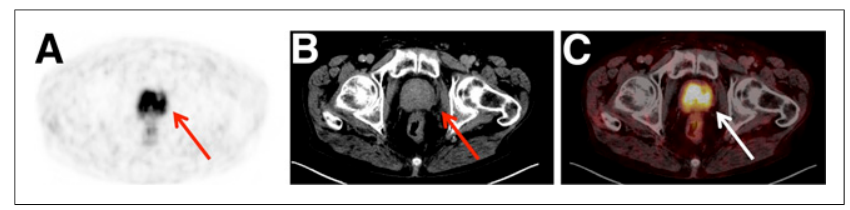

FIGURE 2. SSTR-RADS-1B. Image of patient with increased Chromogranin A levels, referred for initial staging. Axial ${ }^{68} \mathrm{Ga}-D O T A T O C$ PET (A), axial CT (B), and axial ${ }^{68} \mathrm{Ga}-\mathrm{DOTATOC}$ PET/CT (C) demonstrating increased uptake in prostate (arrow), for example, caused by prostatitis or due to benign prostatic hyperplasia. 


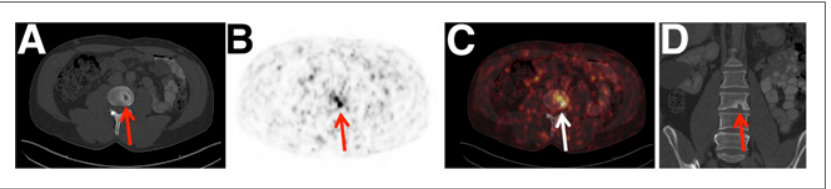

FIGURE 3. SSTR-RADS-2. Likely benign skeletal finding with uptake in patient with NET of pancreatic origin (G1, Ki-67 $=2 \%)$. Axial CT $(A)$, axial ${ }^{68} \mathrm{Ga}-\mathrm{DOTATOC}$ PET (B), axial ${ }^{68} \mathrm{Ga}-\mathrm{DOTATOC}$ PET/CT (C), and coronal CT (D) showing lytic-appearing lesion involving inferior endplate of lumbar vertebral body (arrow). Strongly suspected to be degenerative (a Schmorl's node), this intravertebral disk herniation would be classified as SSTR-RADS-2.

SSTR-RADS-3A signifies level 1-2 uptake in soft-tissue sites typical for NET metastases, such as in regional lymph nodes (Fig. 4), whereas SSTR-RADS-3B refers to level 1-2 uptake in bone lesions not atypical for NET (Fig. 5). In both cases, initial follow-up imaging (SSTR PET or whole-body MRI) might be necessary to confirm definitive diagnosis, although the final interpretation may also depend on Ki-67/grading (21). Similar to PSMA-RADS, we would recommend follow-up imaging after 3-6 mo (3,22); any progression of the lesions would lead to an upgrade to either SSTR-RADS-4 or SSTR-RADS-5 (17). However, the Ki-67 index/ histopathologic grading has to be considered in the context of NET; in the case of an increased proliferation index, follow-up imaging after 3 mo would be preferred. For SSTR-RADS-3B, the treatment algorithm should depend on the biology and number of positive lesions: Single positive lesions might rather be followed or treated with a locoregional procedure, whereas an increased number of growing, SSTR-expressing metastases might guide the treating physician to consider PRRT $(3,23)$.

SSTR-RADS-3C suggests another non-NET malignant process and involves intense uptake (level 3) in a site highly atypical for a NET lesion, for example, radiotracer uptake in the breast (Fig. 6) (20). In many cases, histologic diagnosis (if feasible) can help guide the selection of therapeutic options, as necessary.

SSTR-RADS-3D lesions have a high likelihood for a malignancy (e.g., dedifferentiated NET or other malignant process), but they are negative on an SSTR PET scan. In other words, anatomic findings highly suggestive of being malignant, but demonstrating no SSTR-targeted radiotracer uptake, would be categorized as SSTR-RADS-3D. A common clinical scenario would be dedifferentiation of single NET liver lesions (in a patient with known G2 disease) demonstrating distinct heterogeneous characteristics on a subsequent ${ }^{18}$ F-FDG PET scan (SSTR PET-negative, but ${ }^{18}$ F-FDG PET-positive) (24). Different uptake patterns on ${ }^{68} \mathrm{Ga}-$ DOTATATE and ${ }^{18}$ F-FDG scans normally indicate more aggressive

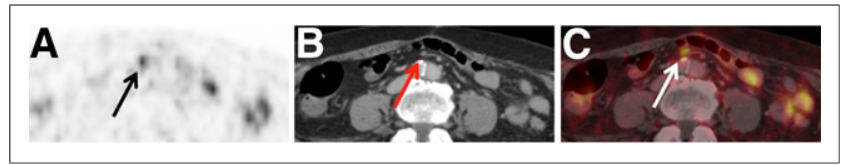

FIGURE 4. SSTR-RADS-3A. Low-level uptake in mesenteric lymph node in midabdomen of patient diagnosed with ileal NET (G1, Ki-67 = $2 \%$ ). Axial ${ }^{68} \mathrm{Ga}$-DOTATOC PET (A), axial CT (B), and axial ${ }^{68} \mathrm{Ga}-\mathrm{DOTA}-$ TOC PET/CT (C) show small (short-axis diameter, $<0.5 \mathrm{~cm}$ ) mesenteric lymph node (arrow). Degree of focal uptake was above blood pool but lower than liver (not shown), and follow-up imaging (after 3 mo) was recommended. Depending on local practice pattern, biopsy might be considered (although biopsy of this site is difficult).

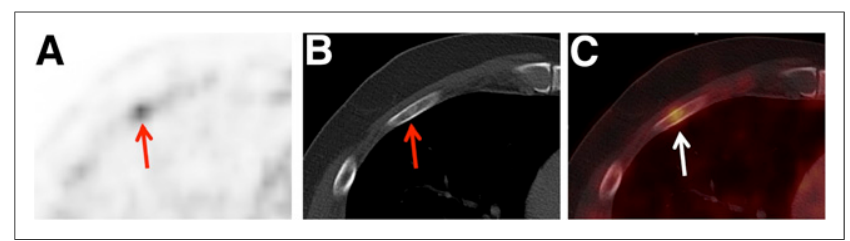

FIGURE 5. SSTR-RADS-3B. Moderate uptake in bone lesion in patient with small bowel NET (G2). Axial ${ }^{68} \mathrm{Ga}$-DOTATOC PET (A), axial CT (B), and axial ${ }^{68} \mathrm{Ga}$-DOTATOC PET/CT (C) show radiotracer uptake in right fifth rib (arrow). Because of CT findings along with moderate uptake on PET, follow-up imaging was recommended.

disease (Fig. 7) (6,25). Hence, in terms of NET lesions suggestive of dedifferentiation, ${ }^{18} \mathrm{~F}$-FDG PET could be performed $(11,26,27)$. Tissue biopsy to confirm the diagnosis and to gain additional prognostic information about the non-SSTR-expressing site of disease may be of value in certain circumstances. PRRT might not be considered, but this depends on grading, overall tumor burden, kidney and bone marrow function, and overall SSTR expression on a whole-patient level. An example would be a G2 NET with most lesions demonstrating SSTR expression, but a single dedifferentiated SSTR-negative, ${ }^{18} \mathrm{~F}-\mathrm{FDG}$-positive lesion. A combined treatment of PRRT together with a locoregional procedure, such as selective internal radiotherapy or transarterial chemoembolization, could be performed $(28,29)$. In some cases, SSTR-RADS3D lesions are indicative of a non-NET malignant process (i.e., a coexisting second primary tumor with or without metastatic disease). If this is suspected, biopsy to establish the identity of the potential second malignancy is crucial to guide further imaging workup and therapy.

\section{SSTR-RADS-4}

SSTR-RADS-4 describes those findings having intense uptake in sites typical for NET lesions, but without definitive findings on conventional imaging. An example would be intense uptake in a locoregional lymph node (level 3), but without confirmatory findings on anatomic imaging (i.e., small lymph node or marrow-based bone lesions, which are nonsuggestive on a CT scan, Fig. 8). Given the high sensitivity and specificity of SSTR-targeted radiotracers, these lesions are likely to represent sites of NET. In brief, the main difference between SSTR-RADS-4 and SSTR-RADS-5 is the lack of anatomic correlate for SSTR-RADS-4 lesions, adding a slight uncertainty to the diagnosis of a NET lesion. Further confirmation by biopsy is generally not necessary unless prognosis or other precision medicine metrics can be gained by analyzing a tissue specimen. That being said, many of these lesions classified as SSTR-RADS-4 would be difficult to biopsy because of small size (e.g., an

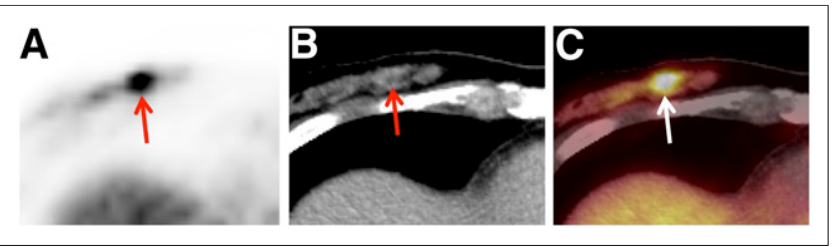

FIGURE 6. SSTR-RADS-3C. Patient with right invasive, lobular breast cancer (pT3, N1, M1 (liver)). Axial ${ }^{68} \mathrm{Ga}-\mathrm{DOTATOC}$ PET (A), axial CT (B), and axial ${ }^{68} \mathrm{Ga}$-DOTATOC PET/CT (C) demonstrating intense uptake in remaining right breast (a site highly atypical for NET lesion) (arrow, level 3). 

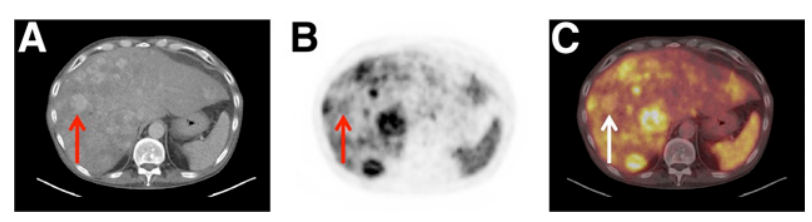

FIGURE 7. SSTR-RADS-3D. Non-radiotracer-avid liver lesion in patient with G2 NET of pancreatic origin with history of cold and hot somatostatin analog treatment (2 cycles of PRRT; cumulative activity, $15.4 \mathrm{GBq}$ of ${ }^{177}$ Lu-DOTATOC). Axial CT (A), axial ${ }^{68} \mathrm{Ga}$-DOTATOC PET (B), and axial ${ }^{68} \mathrm{Ga}-\mathrm{DOTATOC}$ PET/CT (C) show a $2.8-\mathrm{cm}$ hepatic metastasis with negligible uptake above liver background (arrow). ${ }^{18} \mathrm{~F}-\mathrm{FDG}$ PET was recommended to assess underlying intratumoral heterogeneity/ dedifferentiation, with eventual need for PET-guided biopsy being likely.

intensively avid small lymph node that would be difficult to target on CT guidance). On the assumption of a typical distribution of NET, PRRT can be considered $(3,23)$.

\section{SSTR-RADS-5}

SSTR-RADS-5 lesions show intense uptake (level 3) in sites typical for NET and with corresponding findings on conventional imaging (e.g., a liver lesion demonstrating SSTR uptake and typical appearance on CT, Fig. 9). The likelihood of a misdiagnosis is low; hence, similar to PSMA-RADS-5 or BI-RADS-5, biopsy failing to yield a definitive diagnosis has a high risk of being false-negative $(15,17)$. As such, in these lesions, biopsy is unlikely to be of value in confirming the diagnosis, but again may be useful in certain circumstances as described in the SSTR-RADS-4 section. PRRT can definitely be considered $(3,23)$.

\section{DISCUSSION}

In this article, we aimed to establish a standardized reporting system for the clinically most relevant SSTR imaging agents, namely SSTR-RADS version 1.0.

In analogy to PSMA-RADS, reporting on a SSTR PET scan requires a minimum of clinical and imaging acquisition information, which should be mentioned in the clinical report (Supplemental Table 1). If fewer than/equal to 5 positive lesions can be identified, all of these sites should be classified according to the above-mentioned SSTR-RADS classification (along with an anatomic description, maximum diameter for measurable lesions, and expressed mean/ maximum SUV) $(3,11,17)$. The impression could state the SSTRRADS scores for those lesions, but also provide an overall

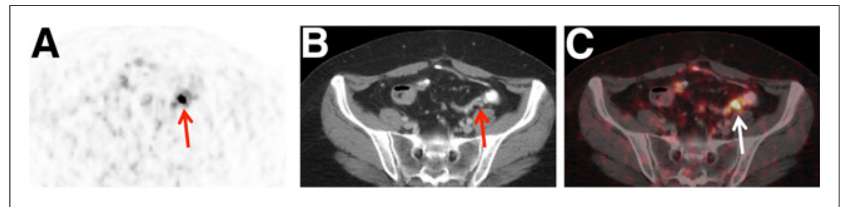

FIGURE 8. SSTR-RADS-4. Patient with ileocecal NET (Ki-67 2\%, G1), with radiotracer-avid lymph node in lower left abdomen that is too small to consider definitively disease-involved on conventional imaging. Axial ${ }^{68} \mathrm{Ga}-\mathrm{DOTATOC}$ PET (A), axial CT (B), and axial ${ }^{68} \mathrm{Ga}-\mathrm{DOTATOC}$ PET/CT (C) images show degree of uptake consistent with metastatic NET lesion (arrow, level 3). However, because short-axis diameter of lymph node was $0.6 \mathrm{~cm}$ (i.e., $<1.0 \mathrm{~cm}$ ), this node would generally not be considered pathologically enlarged on CT.

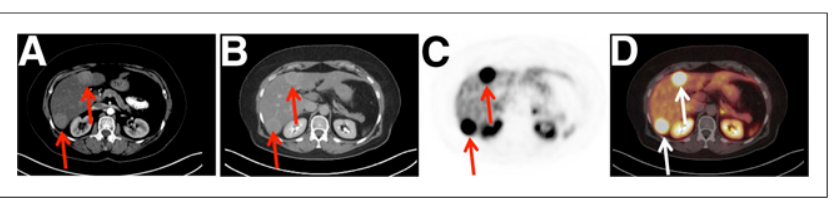

FIGURE 9. SSTR-RADS-5. Image of patient with extensive SSTR-positive liver lesions. Axial CT (A and B), axial ${ }^{68} \mathrm{Ga}$-DOTATOC PET (C), and axial ${ }^{68} \mathrm{Ga}$-DOTATOC PET/CT (D) clearly demonstrating 2 intrahepatic lesions with intense radiotracer uptake (level 3 ) and corresponding findings on CT (arrow). This scan would be categorized as SSTR-RADS-5. PRRT could definitely be considered.

SSTR-RADS score. An overall SSTR-RADS of 3 without mentioning an anatomic description of increased uptake is insufficient, as further workup cannot be undertaken because the exact site of abnormal SSTR expression is still lacking (17). If more than 5 positive lesions can be detected, a dominant, representative lesion per "system" should be chosen (i.e., the largest or hottest lesion, such as primary tumor or hottest lymph node/ organ metastasis); however, contrary to ${ }^{18} \mathrm{~F}$-FDG imaging findings in NET malignancies (30), the role of a dominant/hottest lesion on a SSTR PET has not yet been fully investigated. Nevertheless, the "highest" SSTR-RADS lesion will also designate the overall PET score and might "overrule" "lower" lesions, that is, if 1 lesion is classified as SSTR-RADS-3A or $-3 \mathrm{~B}$, but the remaining lesions are SSTR-RADS-4 or -5 , the findings for SSTR-RADS-3A or -3B lesions can be waived (17). If the overall score is SSTR-RADS-4 or SSTR-RADS-5, PRRT should be considered (Fig. 10) (3,23). Nevertheless, SSTR-RADS-3C and -3D are important categories: SSTR-RADS-3C may trigger immediate further workup, whereas in SSTR-RADS-3D further workup is also required but PRRT might be more widely applicable: if only 1 single dedifferentiated lesion is present, a combined treatment of peptide-based radiotherapy with locoregional procedure

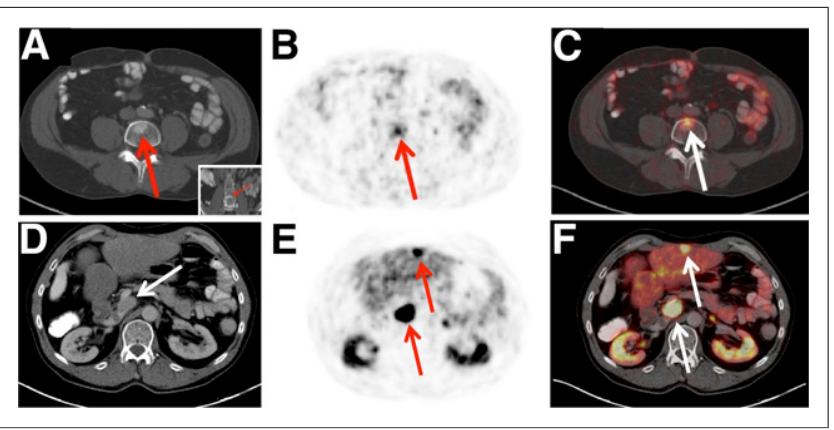

FIGURE 10. Defining overall SSTR-RADS score. Example of patient with colorectal NET (Ki-67 $<2 \%$, G1 NET). Axial/coronal (insert) CT (A), axial ${ }^{68} \mathrm{Ga}-\mathrm{DOTATOC}$ PET (B), and axial ${ }^{68} \mathrm{Ga}-\mathrm{DOTATOC}$ PET/CT (C) reveal equivocal tracer uptake in degenerative, intravertebral disk herniation (most likely a Schmorl's node, SSTR-RADS-2, arrow). However, axial ${ }^{68} \mathrm{Ga}$-DOTATOC PET (E) and axial ${ }^{68} \mathrm{Ga}$-DOTATOC PET/CT (F) clearly demonstrate intense uptake in liver lesion in segment III, which cannot be detected on axial CT (D) (SSTR-RADS-4), highly consistent with liver metastasis. Additionally, intense uptake in pathologically enlarged lymph node close to hilum of liver (arrow) can also be identified on both imaging modalities. As latter would be classified as SSTR-RADS-5, "highest" SSTR-RADS lesion will also designate overall PET score (i.e., SSTR-RADS-5 in this case, "overruling" other lesions). PRRT could be considered. 
might be an option. However, a high tumor burden with several dedifferentiated lesions rules out PRRT (e.g., several SSTR-negative $/{ }^{18} \mathrm{~F}$-FDG-positive liver metastases or several liver metastases that are negative on SSTR PET but are present on MRI) (11). Tumor heterogeneity (SSTR-negative/ ${ }^{18}$ F-FDG-positive lesions) should be reported, and local treatment of SSTR nonavid lesions should be considered $(28,29)$.

Despite the aforementioned goal of increasing the reader's confidence of evaluating SSTR PET scans, the herein proposed SSTRRADS system could be potentially applied in numerous clinical settings. Recent reports have investigated the pretherapeutic uptake of SSTR PET (SUVs) for outcome prediction in patients scheduled for PRRT, using either ${ }^{177} \mathrm{Lu}$ - or ${ }^{90}$ Y-DOTATATE/-TOC (31-33). However, reliable outcome predictors are still intensively sought: the Delphic Consensus Assessment for GEP-NET disease management reported on the limitations of Chromogranin A alterations as well as Ki-67 for identifying potential PRRT candidates (34). Moreover, SSTR-RADS could also be expanded to other SSTR-expressing, non-GEP tumors, such as small cell lung cancer or cancer of unknown primary (35-37). Hence, in light of the increased use of SSTR PET outside of controlled clinical trials, SSTR-RADS could serve as a more sophisticated approach for outcome prediction in PRRT.

While adapting some aspects of the PSMA-RADS system to SSTR molecular imaging, we maintained the initial proposed 5-point RADS scale for prostate molecular imaging: once the underlying framework of PSMA-RADS has been successfully understood (17), it can now be readily applied for SSTR PET using SSTR-RADS, and imaging interpreters who are familiar with one system should be able to learn the other system. Nonetheless, the differences in the underlying biologies of both tumor entities have to be considered while reporting on either PSMA or SSTR PET scans. However, further analysis of interreader agreement is of utmost importance before SSTR-RADS can be implemented in clinical routine.

\section{CONCLUSION}

We herein propose a novel standardization system for SSTR PET scans, which may have utility in identifying potential pitfalls in interpretation and measure the reader's confidence in the presence or SSTR-expressing tumor. This system may also guide the treating physician in selecting PRRT candidates. Further confirmatory work is needed to validate this proposed reporting system. Apart from that, we hope that the vision of SSTR-RADS as a way toward standardization will be pursued in daily clinical routine. SSTR-RADS is subject to continuous development and therefore, we highly appreciate any further input by imaging specialists to improve this scoring system.

\section{DISCLOSURE}

This project has received funding from the European Union's Horizon 2020 research and innovation programme under the Marie Sklodowska-Curie grant agreement No 701983. No other potential conflict of interest relevant to this article was reported.

\section{REFERENCES}

1. Strosberg J, El-Haddad G, Wolin E, et al. Phase 3 trial of ${ }^{177} \mathrm{Lu}$-Dotatate for midgut neuroendocrine tumors. N Engl J Med. 2017;376:125-135.
2. Baum RP, Kluge AW, Kulkarni H, et al. [ ${ }^{177}$ Lu-DOTA](0)-D-Phe(1)-Tyr(3)Octreotide $\left({ }^{177} \mathrm{Lu}\right.$-DOTATOC) for peptide receptor radiotherapy in patients with advanced neuroendocrine tumours: a phase-II study. Theranostics. 2016;6:501510 .

3. Bodei L, Mueller-Brand J, Baum RP, et al. The joint IAEA, EANM, and SNMMI practical guidance on peptide receptor radionuclide therapy (PRRNT) in neuroendocrine tumours. Eur J Nucl Med Mol Imaging. 2013;40:800816.

4. Nicolas GP, Beykan S, Bouterfa H, et al. Safety, biodistribution, and radiation dosimetry of ${ }^{68} \mathrm{Ga}$-OPS202 $\left({ }^{68} \mathrm{Ga}\right.$-NODAGA-JR11) in patients with gastroenteropancreatic neuroendocrine tumors: a prospective phase I imaging study. $\mathrm{J} \mathrm{Nucl}$ Med. October 12, 2017 [Epub ahead of print].

5. Nicolas GP, Schreiter N, Kaul F, et al. Comparison of ${ }^{68} \mathrm{Ga}-\mathrm{OPS} 202\left({ }^{68} \mathrm{Ga}-\right.$ NODAGA-JR11) and ${ }^{68} \mathrm{Ga}$-DOTATOC $\left({ }^{68} \mathrm{Ga}\right.$-Edotreotide) PET/CT in patients with gastroenteropancreatic neuroendocrine tumors: evaluation of sensitivity in a prospective phase II imaging study. J Nucl Med. November 30, 2017 [Epub ahead of print].

6. Kwekkeboom DJ, Kam BL, van Essen M, et al. Somatostatin-receptor-based imaging and therapy of gastroenteropancreatic neuroendocrine tumors. Endocr Relat Cancer. 2010;17:R53-R73.

7. Bodei L, Ambrosini V, Herrmann K, Modlin I. Current concepts in ${ }^{68}$ Ga-DOTATATE imaging of neuroendocrine neoplasms: interpretation, biodistribution, dosimetry, and molecular strategies. J Nucl Med. 2017;58:1718-1726.

8. Shastry M, Kayani I, Wild D, et al. Distribution pattern of ${ }^{68} \mathrm{Ga}$-DOTATATE in disease-free patients. Nucl Med Commun. 2010;31:1025-1032.

9. Kroiss A, Putzer D, Decristoforo C, et al. ${ }^{68}$ Ga-DOTA-TOC uptake in neuroendocrine tumour and healthy tissue: differentiation of physiological uptake and pathological processes in PET/CT. Eur J Nucl Med Mol Imaging. 2013;40: 514-523.

10. Tarkin JM, Joshi FR, Evans NR, et al. Detection of atherosclerotic inflammation by ${ }^{68} \mathrm{Ga}$-DOTATATE PET compared to $\left[{ }^{18} \mathrm{~F}\right] \mathrm{FDG}$ PET imaging. $\mathrm{J}$ Am Coll Cardiol. 2017;69:1774-1791.

11. Bozkurt MF, Virgolini I, Balogova S, et al. Guideline for PET/CT imaging of neuroendocrine neoplasms with ${ }^{68} \mathrm{Ga}$-DOTA-conjugated somatostatin receptor targeting peptides and ${ }^{18}$ F-DOPA. Eur J Nucl Med Mol Imaging. 2017;44: 1588-1601.

12. Fueger BJ, Hamilton G, Raderer M, et al. Effects of chemotherapeutic agents on expression of somatostatin receptors in pancreatic tumor cells. J Nucl Med. 2001; 42:1856-1862.

13. Weinreb JC, Barentsz JO, Choyke PL, et al. PI-RADS prostate imaging: reporting and data system-2015, version 2. Eur Urol. 2016;69:16-40.

14. McKee BJ, Regis SM, McKee AB, Flacke S, Wald C. Performance of ACR Lung-RADS in a clinical CT lung screening program. $J$ Am Coll Radiol. 2016;13:R25-R29.

15. Orel SG, Kay N, Reynolds C, Sullivan DC. BI-RADS categorization as a predictor of malignancy. Radiology. 1999;211:845-850.

16. Grant EG, Tessler FN, Hoang JK, et al. Thyroid ultrasound reporting lexicon: white paper of the ACR thyroid imaging, reporting and data system (TIRADS) committee. J Am Coll Radiol. 2015;12:1272-1279.

17. Rowe SP, Pienta KJ, Pomper MG, Gorin MA. Proposal for a structured reporting system for prostate-specific membrane antigen-targeted PET imaging: PSMARADS version 1.0. J Nucl Med. 2018;59:479-485.

18. Fanti S, Farsad M, Le M. Somatostatin receptor PET-CT. In: PET-CT Beyond FDG: A Quick Guide to Image Interpretation. Berlin, Germany: Springer-Verlag Berlin Heidelberg; 2010:99-123.

19. Hofman MS, Lau WF, Hicks RJ. Somatostatin receptor imaging with ${ }^{68} \mathrm{Ga}$ DOTATATE PET/CT: clinical utility, normal patterns, pearls, and pitfalls in interpretation. Radiographics. 2015;35:500-516.

20. Naswa N, Sharma P, Kumar R, Malhotra A, Bal C. Usual and unusual neuroendocrine tumor metastases on ${ }^{68} \mathrm{Ga}$-DOTANOC PET/CT: a pictorial review. Clin Nucl Med. 2013;38:e239-e245.

21. Schraml C, Schwenzer NF, Sperling O, et al. Staging of neuroendocrine tumours: comparison of $\left[{ }^{68} \mathrm{Ga}\right]$ DOTATOC multiphase PET/CT and whole-body MRI. Cancer Imaging. 2013;13:63-72.

22. Arnold R, Chen YJ, Costa F, et al. ENETS consensus guidelines for the standards of care in neuroendocrine tumors: follow-up and documentation. Neuroendocrinology. 2009;90:227-233.

23. Hicks RJ, Kwekkeboom DJ, Krenning E, et al. ENETS consensus guidelines for the standards of care in neuroendocrine neoplasia: peptide receptor radionuclide therapy with radiolabeled somatostatin analogues. Neuroendocrinology. 2017; 105:295-309.

24. Lapa C, Werner RA, Herrmann K. Visualization of tumor heterogeneity in neuroendocrine tumors by positron emission tomography. Endocrine. 2016;51: $556-557$. 
25. Kuyumcu S, Ozkan ZG, Sanli Y, et al. Physiological and tumoral uptake of ${ }^{68} \mathrm{Ga}-$ DOTATATE: standardized uptake values and challenges in interpretation. Ann Nucl Med. 2013;27:538-545.

26. Kayani I, Bomanji JB, Groves A, et al. Functional imaging of neuroendocrine tumors with combined PET/CT using ${ }^{68} \mathrm{Ga}$-DOTATATE (DOTA-DPhe1,Tyr3octreotate) and ${ }^{18}$ F-FDG. Cancer. 2008;112:2447-2455.

27. Hope TA, Bergsland E, Bozkurt MF, et al. Appropriate use criteria for somatostatin receptor PET imaging in neuroendocrine tumors. J Nucl Med. 2018;59:66-74.

28. Barbier CE, Garske-Roman U, Sandstrom M, Nyman R, Granberg D. Selective internal radiation therapy in patients with progressive neuroendocrine liver metastases. Eur J Nucl Med Mol Imaging. 2016;43:1425-1431.

29. de Mestier L, Zappa M, Hentic O, Vilgrain V, Ruszniewski P. Liver transarterial embolizations in metastatic neuroendocrine tumors. Rev Endocr Metab Disord. 2017;18:459-471.

30. Werner RA, Schmid JS, Higuchi T, et al. Predictive value of FDG-PET in patients with advanced medullary thyroid carcinoma treated with vandetanib. J Nucl Med. 2018;59:756-761.

31. Kratochwil C, Stefanova M, Mavriopoulou E, et al. SUV of $\left[{ }^{68} \mathrm{Ga}\right]$ DOTATOCPET/CT predicts response probability of PRRT in neuroendocrine tumors. Mol Imaging Biol. 2015;17:313-318.
32. Gabriel M, Oberauer A, Dobrozemsky G, et al. ${ }^{68}$ Ga-DOTA-Tyr3-octreotide PET for assessing response to somatostatin-receptor-mediated radionuclide therapy. J Nucl Med. 2009;50:1427-1434.

33. Haug AR, Auernhammer CJ, Wangler B, et al. ${ }^{68}$ Ga-DOTATATE PET/CT for the early prediction of response to somatostatin receptor-mediated radionuclide therapy in patients with well-differentiated neuroendocrine tumors. J Nucl Med. 2010;51:1349-1356.

34. Oberg K, Krenning E, Sundin A, et al. A Delphic consensus assessment: imaging and biomarkers in gastroenteropancreatic neuroendocrine tumor disease management. Endocr Connect. 2016;5:174-187.

35. Lapa C, Hanscheid H, Wild V, et al. Somatostatin receptor expression in small cell lung cancer as a prognostic marker and a target for peptide receptor radionuclide therapy. Oncotarget. 2016;7:20033-20040.

36. Sabet A, Haug AR, Eiden C, et al. Efficacy of peptide receptor radionuclide therapy with ${ }^{177} \mathrm{Lu}$-octreotate in metastatic pulmonary neuroendocrine tumors: a dual-centre analysis. Am J Nucl Med Mol Imaging. 2017;7: 74-83.

37. Sharma N, Naraev BG, Engelman EG, et al. Peptide receptor tadionuclide therapy outcomes in a North American cohort with metastatic well-differentiated neuroendocrine tumors. Pancreas. 2017;46:151-156. 